\title{
MEDNIK syndrome
}

INSERM

\section{Source}

INSERM. (1999). Orphanet: an online rare disease and orphan drug data base. MEDNIK syndrome. ORPHA:171851

MEDNIK syndrome, previously known as Erythrokeratodermia Variabilis type 3 (EKV3), is characterized by intellectual deficit, enteropathy, sensorineural hearing loss, peripheral neuropathy, lamellar and erythrodermic ichthyosis, and keratodermia (MEDNIK stands for Mental retardation, Enteropathy, Deafness, peripheral Neuropathy, Ichtyosis, Keratodermia). 